Fissile and Non-Fissile Material Detection using Nuclear Acoustic Resonance Signatures: Final Report

J. Herberg, R. Maxwell, B. R. Tittmann, P. M. Lenahan, S. Yerkes, S. B. Jayaraman

November 10, 2006 
This document was prepared as an account of work sponsored by an agency of the United States Government. Neither the United States Government nor the University of California nor any of their employees, makes any warranty, express or implied, or assumes any legal liability or responsibility for the accuracy, completeness, or usefulness of any information, apparatus, product, or process disclosed, or represents that its use would not infringe privately owned rights. Reference herein to any specific commercial product, process, or service by trade name, trademark, manufacturer, or otherwise, does not necessarily constitute or imply its endorsement, recommendation, or favoring by the United States Government or the University of California. The views and opinions of authors expressed herein do not necessarily state or reflect those of the United States Government or the University of California, and shall not be used for advertising or product endorsement purposes.

This work was performed under the auspices of the U.S. Department of Energy by University of California, Lawrence Livermore National Laboratory under Contract W-7405-Eng-48. 
UCRL-TR-

\title{
Fissile and Non-Fissile Material Detection using Nuclear Acoustic Resonance Signatures: Final Report
}

\author{
Julie Herberg, Robert Maxwell \\ Center for National Security Applications of Magnetic Resonance \\ CMLS and Engineering Directorates \\ Lawrence Livermore National Laboratory \\ Livermore, CA 94550
}

Prof. Bernhard R. Tittmann, Prof. Pat M. Lenahan, Shane Yerkes, and Subash B. Jayaraman

Department of Engineering Science and Mechanics

The Pennsylvania State University

University Park, PA

October 31, 2006 


\section{Executive Summary}

This is final report on NA-22 project LL251DP, where the goal was to develop a novel technique, Nuclear Acoustic Resonance (NAR), for remote, non-destructive, nonradiation-based detection of materials of interest to Nonproliferation Programs, including ${ }^{235} \mathrm{U}$ and ${ }^{239} \mathrm{Pu}$. In short, we have developed a magnetic shield chamber and magnetic field, develop a digital lock-in amplifier computer to integrate both the ultrasound radiation with the detector, developed strain measurements, and begun to perform initial measurements to obtain a NAR signal from aluminum at room temperature and near the earth's magnetic field. Since our funding was cut in FY06, I will discuss where this project can go in the future with this technology.

\section{Introduction:}

We were developing a novel technique, Nuclear Acoustic Resonance (NAR), for remote, non-destructive, non-radiation-based detection of materials of interest to Nonproliferation Programs, including ${ }^{235} \mathrm{U}$ and ${ }^{239} \mathrm{Pu}$. This new technique uses the unique NAR signatures generated by acoustic excitation of phonon modes via ultrasound. The acoustic modulation of internal interactions involving the magnetic and electric multipole moments of the nucleus is potentially observable by nuclear or electron spin transitions. Our approach takes advantage of collaboration with The Pennsylvania State University (PSU), which has expertise in ultrasound and electron spin resonance, along with LLNL's expertise in Nuclear Magnetic Resonance (NMR). The current collaboration between PSU and LLNL will focus on producing a definitive initial estimate of the advantages and limitations of this approach for materials containing ${ }^{235} \mathrm{U}$ and ${ }^{239} \mathrm{Pu}$. If successful, the project will be transferred to LLNL, where NAR techniques will be performed on actual samples of special nuclear materials. We were in the final stages of estimating the advantage and limitations of this approach and plan to conclude this phase of the project in FY06. However, due to the lack of funding we were unable to finish this evaluation. Since this technique does not rely on nuclear radiation detection, the eventual device objective would be to detect and identify fissile materials even if steps had been taken by smugglers to completely shield the materials as a radiation source. A successful device could have enormous potential for the detection and identification of concealed fissile materials, a key and still unresolved issue facing nonproliferation and counterterrorism.

\section{Progress:}

In this report, we are addressing major achievements in developing an NAR experiment for remote, non-destructive, non-radiation-based detection of materials of interest to Nonproliferation Programs, which include:

- developing a magnetic shield chamber and magnetic field to cut down on background noise and slightly increase the earth's magnetic field (Appendix A)

- developing a digital lock-in amplifier computer to integrate both the ultrasound radiation with the detector (Appendix B)

- performed strain measurements to determine the amount of acoustic wave we need to drive the electronic and nuclear interactions. (Appendix $C$ ) 
- begun to perform initial measurements to obtain an NAR signal for aluminum at room temperature and near the earth's magnetic field. (Appendix D)

- discussion of future experiments to probe the feasibility of NAR for detection of ${ }^{235} \mathrm{U}$ and ${ }^{239} \mathrm{Pu}$. (Appendix E)

\section{Outlook / Conclusions:}

In FY05, we constructed a magnetic shield chamber and magnetic field to cut down on background noise and slightly increase the earth's magnetic field and got the potassium magnetometer up and running. In addition, we have performed strain measurements to determine the amount of acoustic wave we need to drive the electronic and nuclear interactions. This has all led to developing the initial measurements to obtain an NAR signal for aluminum at room temperature and near the earth's magnetic field. Even though the funding for this project was cut in FY06, the concept of NAR applied to nuclear proliferation is not only an interesting problem; but could be extremely effective. In order to show the effectiveness of this technique, a demonstration of NAR on ${ }^{235} \mathrm{U}$ and/or ${ }^{239} \mathrm{Pu}$ would need to be performed at $77 \mathrm{~K}$ and in at least a $2 \mathrm{~T}$ magnetic field. 


\section{Appendix A: Developing a magnetic shield chamber and magnetic field to cut down on background noise and slightly increase the earth's magnetic field}

We have determined that both the Overhauser and potassium magnetometer require a very stable and homogeneous field in order to minimize the background noise. We have researched magnetically shielded chambers and carried out fairly extensive calculations on the (very highly uniform) magnetic fields which can be generated within a long cylindrical high permeability chamber with a solenoid. With the help of Ammuneal Corp., we have developed and obtained a three-walled magnetically shielded chamber, which is shown in Figure 1. Students at PSU in Professor Lenahan's group have designed and constructed a simple, relatively inexpensive temperature compensated Hall Effect probes to monitor the small ambient AC and DC magnetic fields outside the magnetically shielded chamber. Students are also working on a magnetic field controller for the shielded chamber/solenoid/magnetometer system. These students have also built a large solenoid, which is shown in Figure 2, to produce an extremely uniform static magnetic field with a field strength of 1.78 Gauss. Along with this large solenoid, these students have designed and built a circuit to provide a steady current supply for the uniform magnetic field.

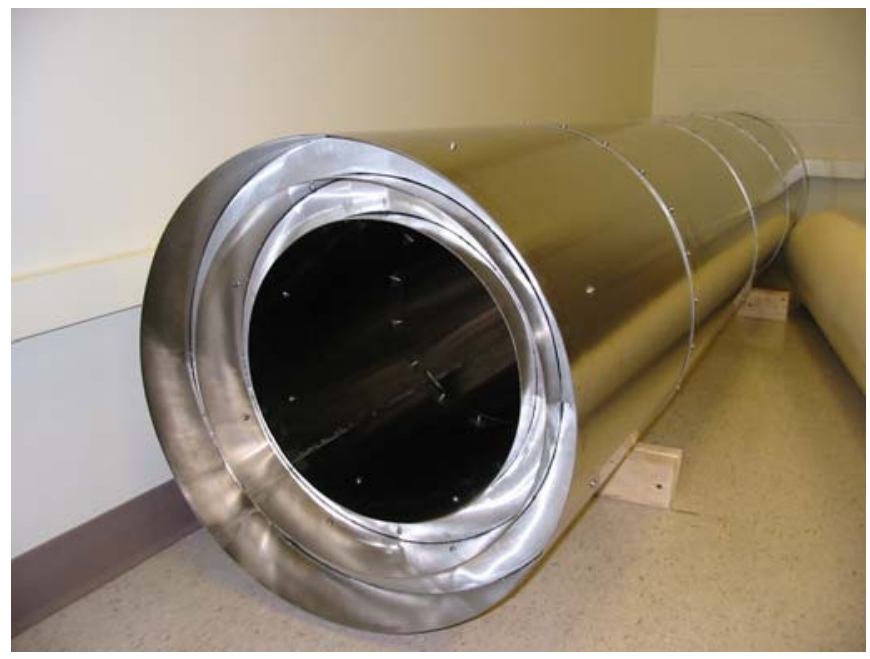

Figure 1: Triple magnetically shielded chamber.

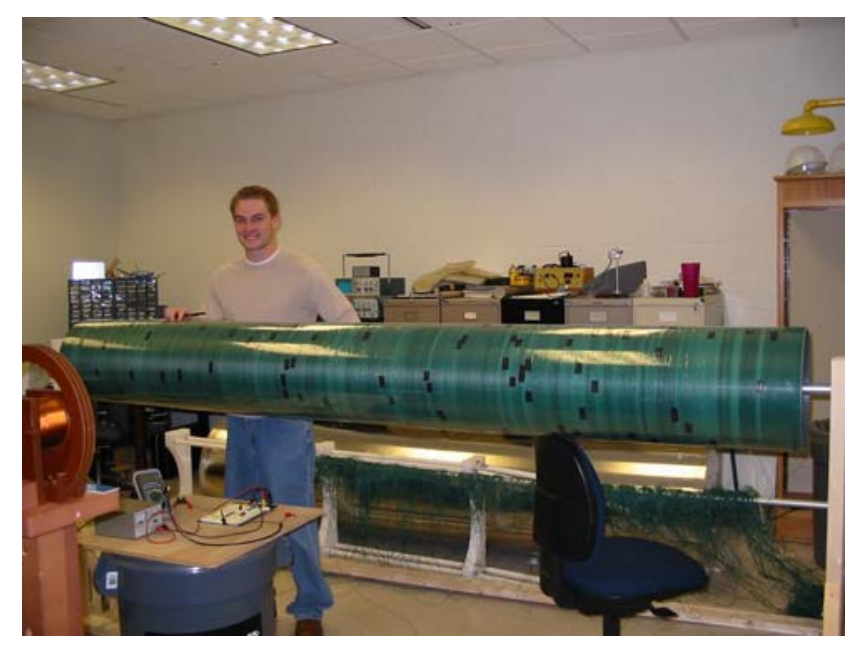

Figure 2: Extremely uniform static magnetic field with a magnetic field of 1.78 Gauss. 


\section{Appendix B: Developing a digital lock-in amplifier computer to integrate both the ultrasound radiation with the detector}

A digital lock-in amplifier has been implemented in software using the Lab View environment. This software controlled lock-in amplifier has many advantages over the traditional lock-in amplifier equipment for our acoustic magnetic resonance applications. First of all, this system is portable. Only a computer and a Data Acquisition System (DAQ) are required for operation. Secondly, a Graphical User Interface (GUI) was created for easy use. With the incorporation of a GUI, the user can adjust the modulation frequency, the modulation amplitude, the modulation phase, the bandpass filter width, and the time constant of the low pass filter. Also, the signal amplitude and xy plot are implemented for visual feedback. The GUI can be seen in Figure 3. A third advantage of this software controlled lock-in amplifier is that settings can be saved to the computer for multiple users. This is useful for the case where multiple experiments are performed using the same acquisition system.

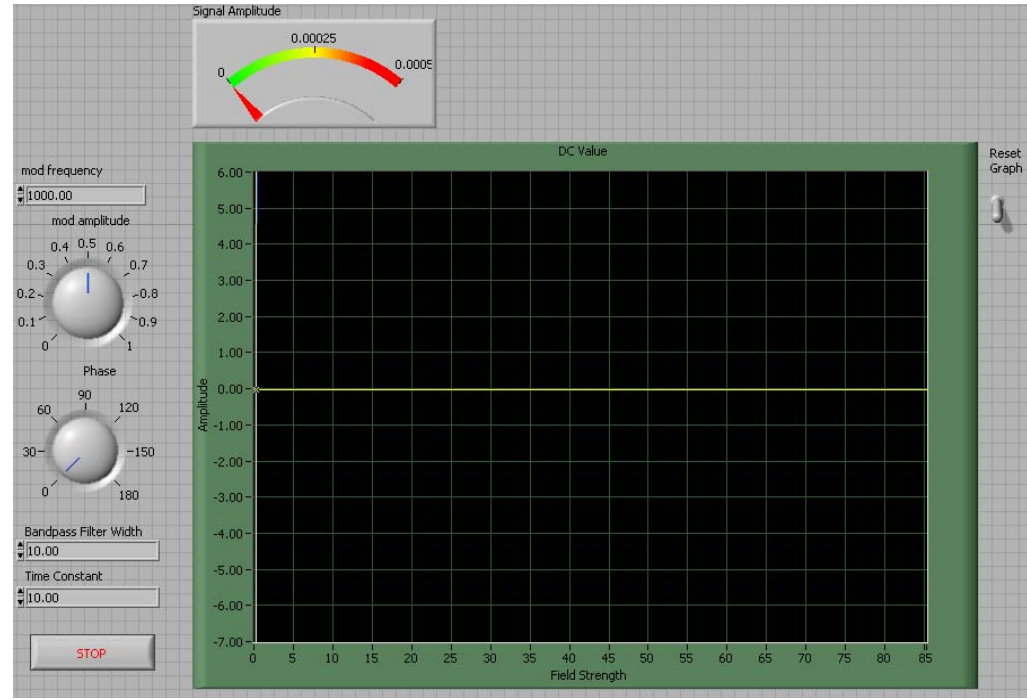

Figure 3: Graphical User Interface of the Digital Lock-in Amplifier

The basic block diagram that represents the function of the digital lock-in can be seen in Figure 4. The main components of the lock-in amplifier are the voltage controlled oscillator, AC amplifier, a multiplier, a low pass filter, and a DC amplifier. All of these functions are simulated and controlled digitally through Lab View. 


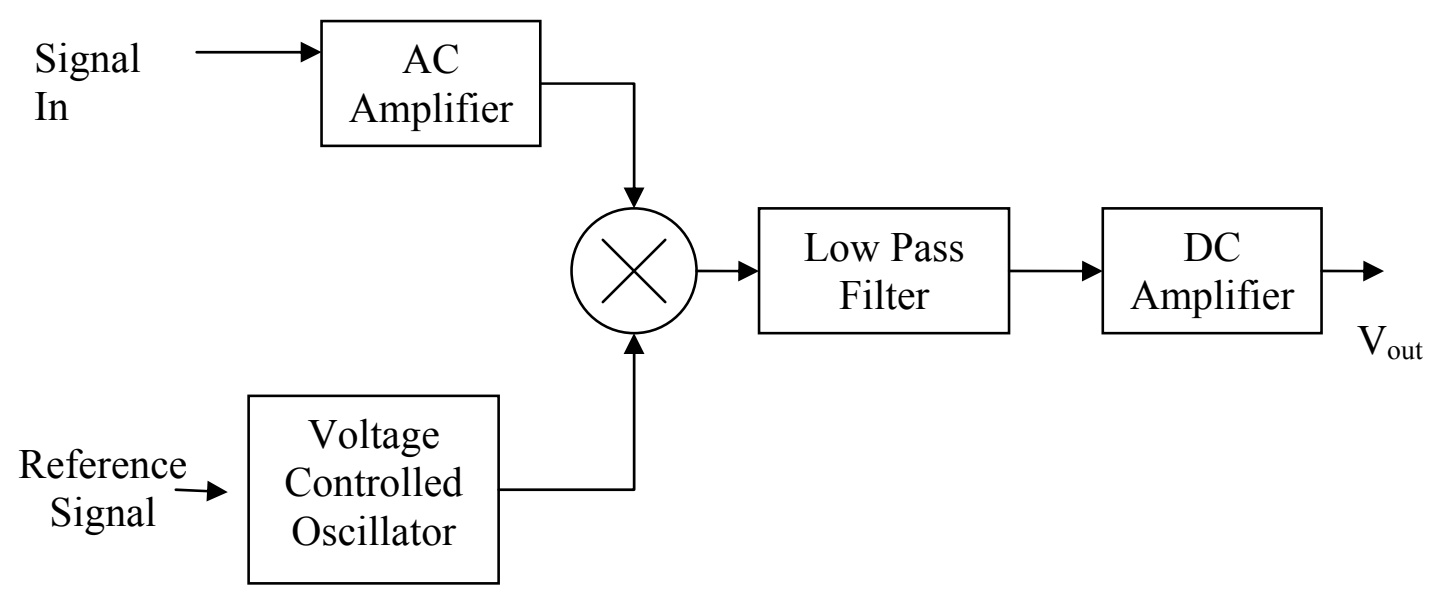

Figure 4: This figure represents the block diagram of a traditional lock-in.

In summary, a digital lock-in amplifier is more advantageous than the traditional lock-in amplifiers. This lock-in amplifier is extremely useful and easy to use; it can provide data acquisition at essentially arbitrary modulation frequencies and time constants, making it particularly useful for our acoustic magnetic resonance studies. 


\section{Appendix C: Performed strain measurements to determine the amount of acoustic wave we need to drive the electronic and nuclear interactions}

Experiments were conducted at Penn State by Prof. Tittmann and Subash Jayaraman, to measure the amount of strain being imparted to a polycrystalline Aluminum cylinder (10 cm long, $3.2 \mathrm{~cm}$ in diameter), using piezoelectric transducers. A Polytec ${ }^{\circledR}$ OFV-505 Laser Doppler Vibrometer (LDV) was used to observe the displacements at the cylinder face. Information regarding the vibrometer principles and operation could be found at www.polytec.com.

From ENDOR tables, the nuclear and electron spin resonance frequencies for Aluminum, in earth's magnetic field $(0.545 \mathrm{G})$, were calculated to be $599.57 \mathrm{~Hz}$ and $1.511 \mathrm{MHz}$ respectively.
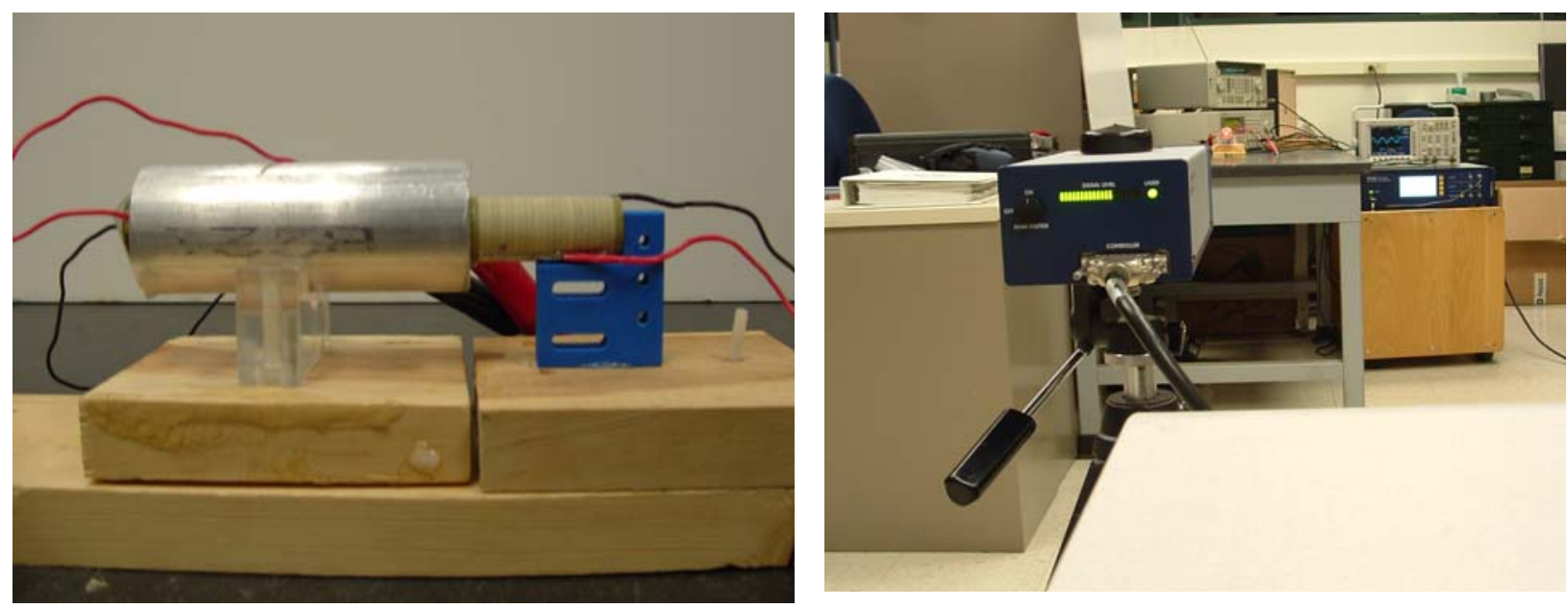

Figure 5. (a) Close-up of the set-up (b) Overall view of the experimental setup

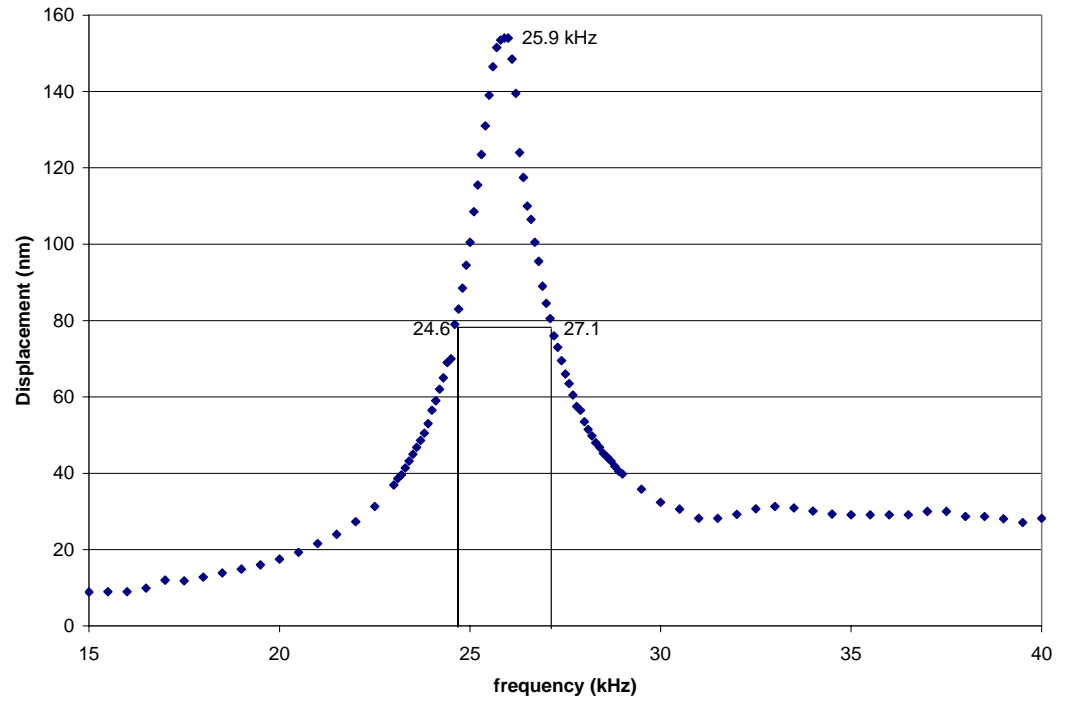

Figure 6. Frequency vs. Displacement curve for the Polycrystalline Aluminum cylinder
The resonant frequency for the Aluminum cylinder (speed of sound in Aluminum is 6400 $\mathrm{m} / \mathrm{s}$ ) is $32 \mathrm{kHz}$. It was decided to combine $599.57 \mathrm{~Hz}$ and $32 \mathrm{KHz}$ frequencies into amplitude modulated (AM) signal and use a 32 $\mathrm{kHz} \quad \mathrm{PZT}$ (leadzirconium-titanate, a piezoelectric material) transducer to excite nuclear spin resonance 
and a $1.51 \mathrm{MHz}$ transducer to excite electron spin resonance. A Wavetek signal generator was used to drive the transducers. Honey was used as the ultrasonic couplant in the experiments.

Figure 5 shows the experimental set up with the transducers attached to the Aluminum specimen. Initial experiments involved vibrating the cylinder about the resonant frequency $(32 \mathrm{kHz})$ and observing the displacements. The input voltage was 10 $\mathrm{V}$, peak-to-peak. It was observed that the peak displacement was observed at $25.9 \mathrm{kHz}$, instead of the expected $32 \mathrm{kHz}$. This is due to the fact that when the transducer is attached to the cylinder, the composite resonating length is increased and hence, there is a reduction in the resonant frequency. The displacements were observed while performing a frequency sweep about the harmonic frequency (Figure 6).

The Quality-factor (Q-factor) is given by $f / \Delta f$, where $f$ is the frequency at which the peak displacement is observed (resonant frequency) and $\Delta \mathrm{f}$ is the bandwidth at which the displacements are $50 \%$ of the peak value. The Q-factor indicates how well the sound is transmitted into the specimen. For the current set-up, it was determined to be approximately 10 . This is a very low value. To improve the Q-factor, a 3-point holder was designed and used to hold the specimen, which improved it to 16. Further efforts are underway to improve the Q-factor such as a better holder for the transducer etc. Peak displacement of $154 \mathrm{~nm}$ was observed which equates to an observed strain of 1.54 microstrains. Strains as low as 0.1 microstrains could be observed as well using the LDV.

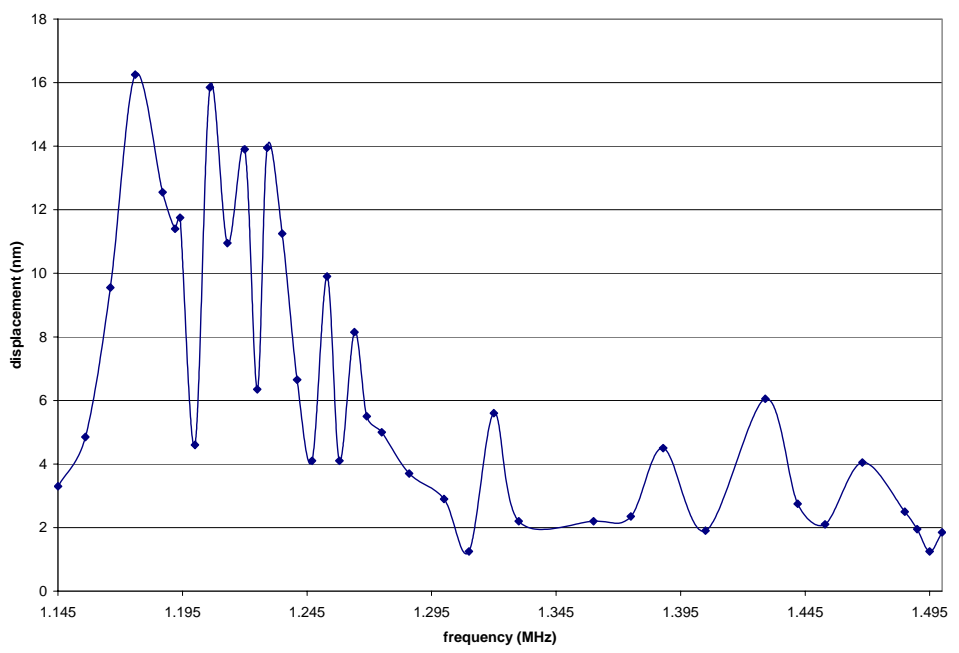

Figure 7: Frequency vs. Displacement-- $1.51 \mathrm{MHz}$ transducer attached to the cylinder 
When vibrating the cylinder with $1.511 \mathrm{MHz}$ transducer, it was very difficult to observe the displacements as $1.511 \mathrm{MHz}$ is much higher harmonic and at this frequency many different modes are also excited. This leads to not obtaining a clear frequency vs. displacement curve (Figure 7).

To identify the Q-factor of the $1.51 \mathrm{MHz}$ transducer, a thin aluminum foil was attached to the transducer and the resultant displacements were observed (Figure 8). The Q-factor was estimated to be 95 . It can be noticed that the displacements generated by the $1.51 \mathrm{MHz}$ transducer are much smaller than the $32 \mathrm{kHz}$ transducer.

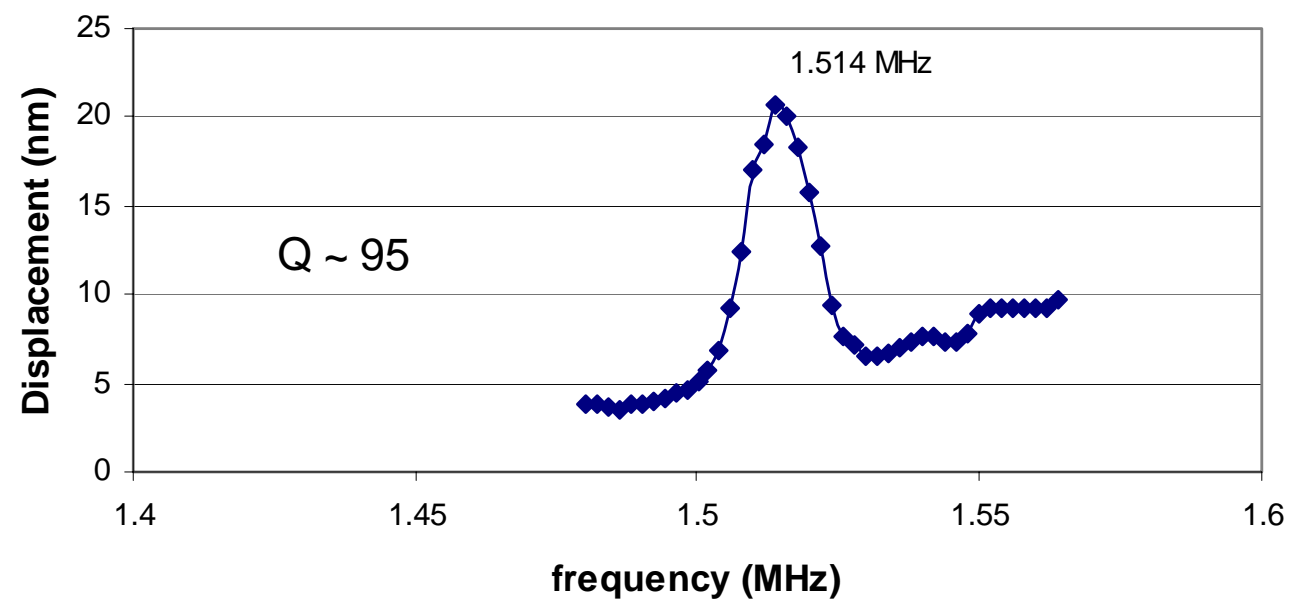

Figure 8. Q-Factor for 1.51 MHz with an aluminum foil attached to the transducer

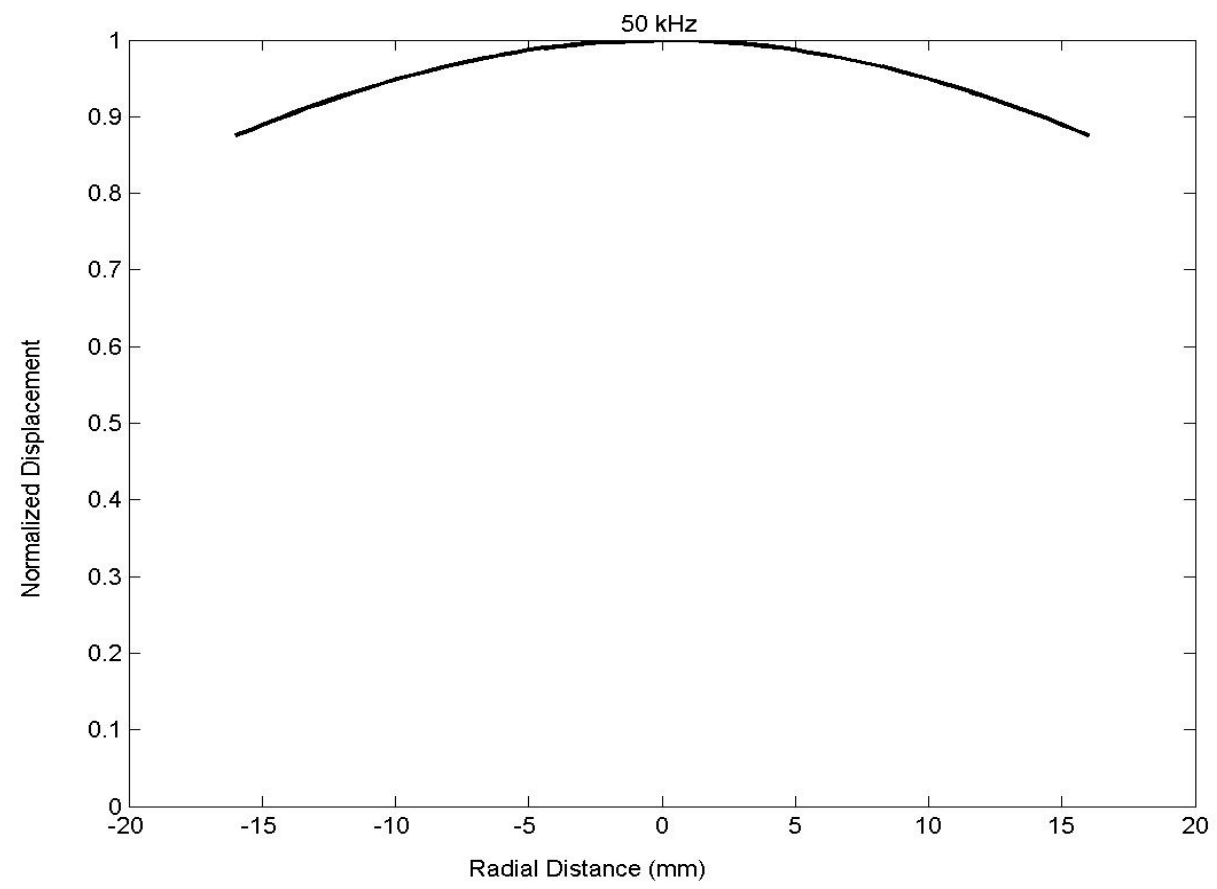

Figure 9. Simulated radial distance vs. displacement for $50 \mathrm{kHz}$ excitation 
The aluminum cylinder was vibrated using the AM signal as well and the components of the LDV signal were separated (namely in to $599 \mathrm{~Hz}$ and $32 \mathrm{kHz}$ ) and analyzed. They produced displacements similar to that shown in figure 7. An online MATLAB code, which simulates the displacements due to longitudinal vibrations in cylinders, was used to compare with the experimental data. The code helps to plot the variation in the displacements along the face of the cylinder. It was observed that, since the $32 \mathrm{kHz}$ PZT transducer was smaller than the cylinder's diameter, there was a large difference between the displacements at the outer radius and at the center of the cylinder. To validate the code, a different transducer $(50 \mathrm{kHz})$ with diameter slightly larger than the cylinder was used. These results were similar which provides confidence in the code. Those results are provided in the Figures 9-11.

Two power amplifiers have been procured which will help us in increasing the amount of strains imparted to the specimen. Efforts are underway to exactly calculate the strain energy imparted to the specimen and also understand the amount of energy required to cause the nuclear spin resonance due to the Alpher-Rubin mechanism (Dipole-dipole interaction) in aluminum.

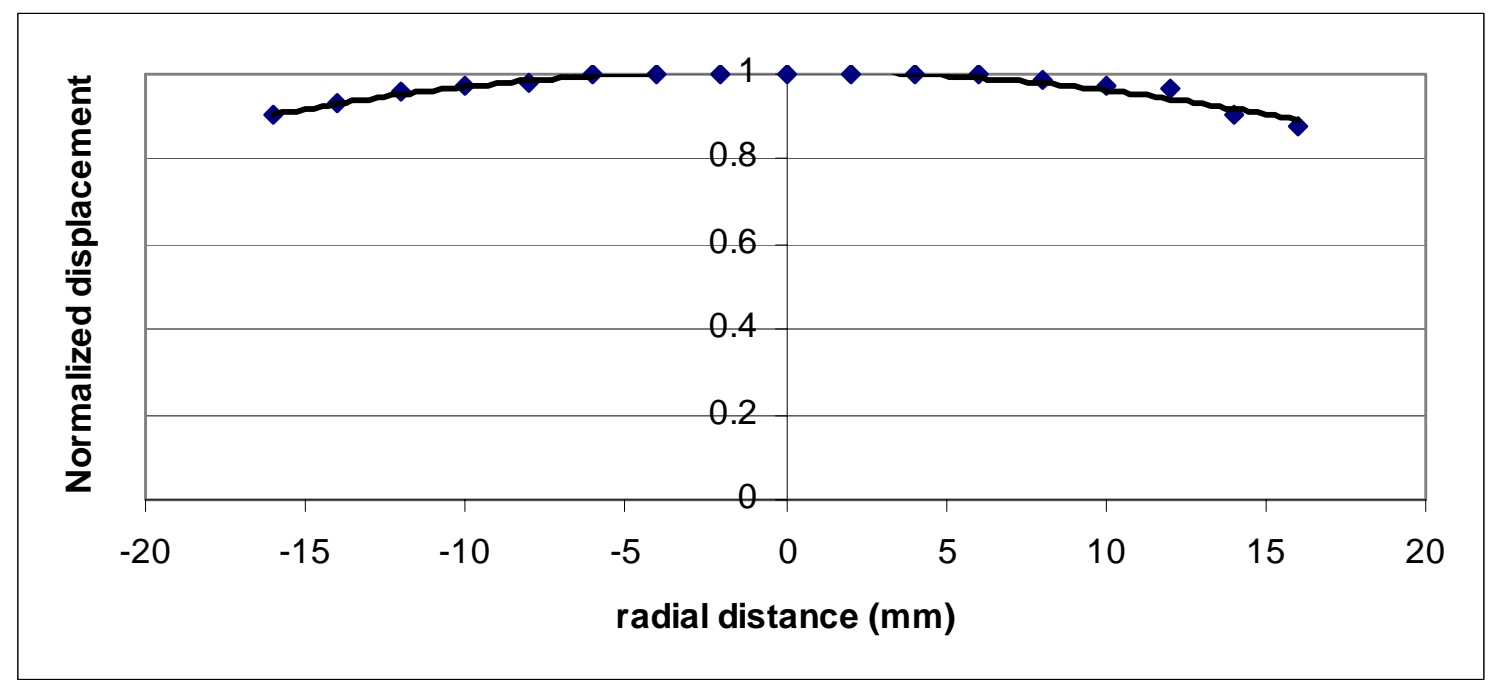

Figure 10. Experimental radial distance vs. displacement for $50 \mathrm{kHz}$ excitation

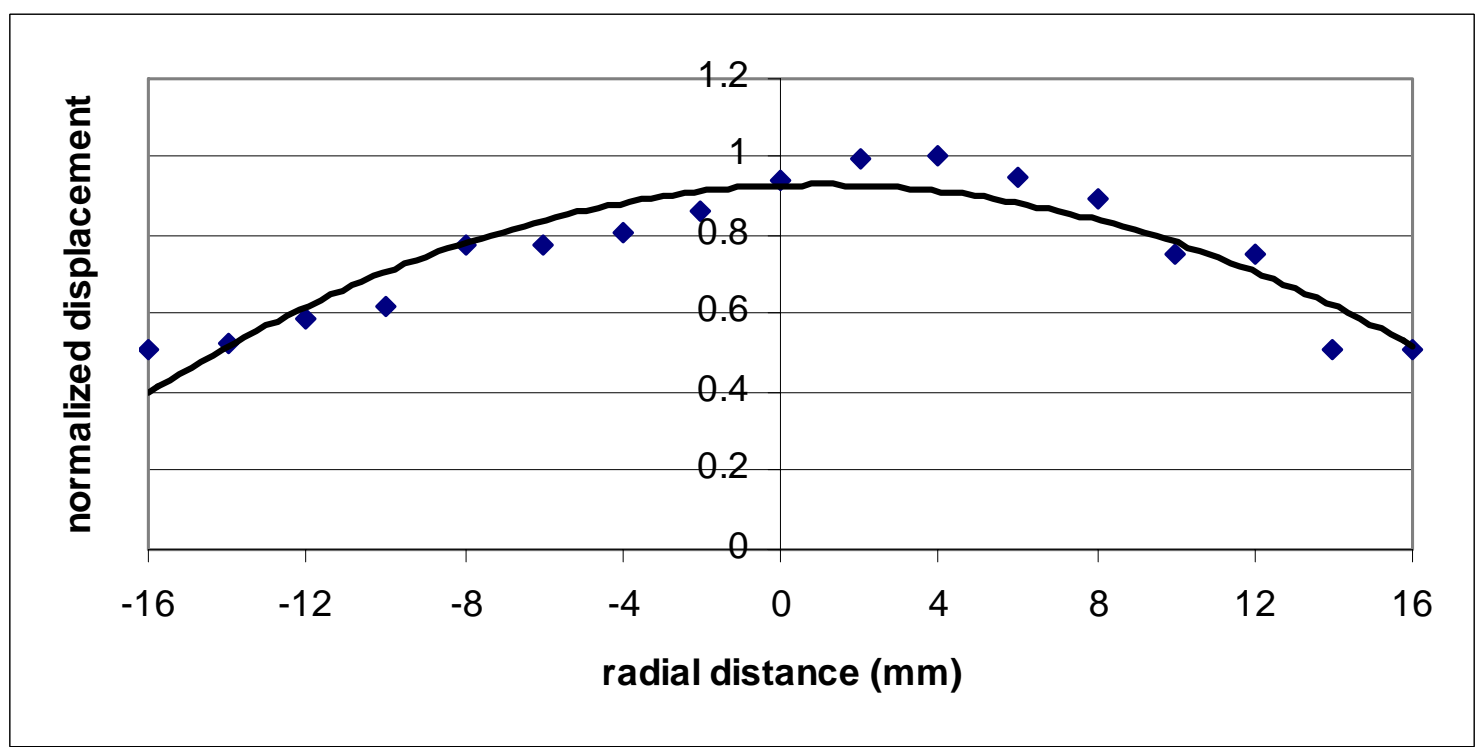

Figure 11. Experimental radial distance vs. displacement for $32 \mathrm{kHz}$ excitation 


\section{Appendix D: Performed initial measurements to obtain an NAR signal for aluminum at room temperature and near the earth's magnetic field.}

Our first attempts at monitoring changes in magnetization brought about by saturating the electron and nuclear spin systems of a sample of aluminum with acoustic waves have been unsuccessful. This null result is likely due to either (1) the fact that we may only be saturating a small portion of the rather large electron spin linewidth of aluminum, or (2) the amplitude of the atomic displacement caused by the acoustic waves is not large enough to create a sufficient $B_{0}$ field.

Over the past few weeks, our efforts have been focused on demonstrating nuclear acoustic resonance (NAR) on a "simpler" sample. Dr. Ivan Hrovic, the president of GEM Systems, Inc. (the manufacturer of our potassium magnetometer), recommended that a solution of TEMPO (2,2,6,6-Tetramethylpiperidine 1-oxyl) and water would be a good system for us to start with. GEM Systems implements the Overhauser effect on a similar nitroxide at Earth's field in one of their other magnetometers. Such nitroxides offer very narrow ESR line widths and the possibility of improving on the Overhauser enhancement factor (theoretical nuclear polarization factors higher than 2000 are possible in low magnetic fields such as Earth's field).

We have made $T_{1}$ measurements on solutions with varying concentrations of TEMPO to ensure that the spin-spin relaxation time of the protons is not shortened too much (by the addition of the TEMPO) to prevent the Overhauser effect from working. At a concentration of $250 \mathrm{mg}$ of TEMPO per liter of water, $\mathrm{T}_{1}$ is only shortened to 1.35 seconds (compared to 3.6 seconds for pure de-ionized water).

Unfortunately, due to the strong hyperfine interaction of the free electron with the nitrogen nucleus, the expression for calculating the electron spin resonance (ESR) frequency of TEMPO at high fields is not valid at Earth's field. By comparing TEMPO to other similar nitroxides, we estimate the ESR frequencies to be approximately 65 MHz. In order to determine the exact frequencies needed, we have designed a "probe" consisting of a large rf coil and circuit; tunable within the range of 60-75 MHz. This method will not only determine the exact ESR frequencies, but it will prove that we will indeed be able to detect the small change in magnetization of the sample caused by saturating the spin system. 


\section{Appendix E: Discussion of future experiments to probe the feasibility of NAR for detection of ${ }^{235} \mathrm{U}$ and ${ }^{239} \mathrm{Pu}$.}

Nuclear Acoustic Resonance (NAR), could provide insight into the properties of actinide materials, including ${ }^{235} \mathrm{U}$ and ${ }^{239} \mathrm{Pu}$. The approach would be non-intrusive, highly penetrating, and applicable to all materials of interest for Nonproliferation, and be able to identify and characterize both acoustic and magnetic anomalies in specific nuclear samples, including the PuCOGa $5,{ }^{235} \mathrm{U}$, and ${ }^{239} \mathrm{Pu}$. NAR is similar to Nuclear Magnetic Resonance (NMR) in that it induces transitions at resonances between energy levels that are defined by the interactions of electric and magnetic properties of nuclei with internal and external magnetic fields, electric quadrupole moments, and electric field gradients. Unlike NMR, which utilizes RF radiation, NAR uses acoustic radiation to induce transitions between energy levels. The acoustic modulation of internal interactions involving the magnetic and electric multipole moments of the nucleus is potentially observable by nuclear or electron spin transitions. NAR has the potential for being a nondestructive, highly-penetrating method to detect and identify smuggled actinides and be much more effective then other techniques, such as Muon detection. However, the potential application is an underdeveloped technology and far away from being field deployable. Performing NAR in the laboratory in a high field at liquid nitrogen temperature would not only allow us to determine if this technology could be used for remote detection, but it could also provide insight into important materials, such as the $\mathrm{PuCOGa}_{5}[1]$. NAR will allow us to make a correlation between acoustic vibrations and nuclear electric quadrupole interactions or electric field gradients in a metal, semiconductor or insulator.

The development of NAR technology will potentially lead to a novel device that can be used to detect and identify smuggled materials that might be used in a weapon of mass destruction. All current nuclear-material detection systems utilize technologies that rely on radiation. Such systems have been quite successful at detecting embedded sources, such as plutonium, that emit very energetic nuclear signatures. Unfortunately, radiation signals from uranium sources, including highly enriched uranium, are not as energetic and can be reduced to background levels by shielding with materials such as aluminum. [2,3] An NAR based detector would be a valuable complement to the existing array of radiation-based detection systems. The main advantage over other techniques is that it is noninvasive and non-radiation based. Since no radiation signals are needed for the technique to work, the NAR approach could in principle also detect nonfissile materials of interest to nonproliferation. In addition, NAR is a highly sensitive technique and the technology that it is built upon has been shown to be able to detect extremely minute changes in material properties in a wide range of scientific disciplines. NAR is also highly penetrating. It has the potential to probe things deeply buried into solid or liquid material structures, including metals and alloys, a key attribute desired for detecting and identifying objects hidden inside a thick metal box. While this NAR apparatus could be used in the detection of fissile and non-fissile materials of interest to Nonproliferation, this technique could also lead to development of a noninvasive tool to investigate the quality of the material, such as ${ }^{235} \mathrm{U}$ and ${ }^{239} \mathrm{Pu}$, in an intact radioactive 
apparatus or individual components of composite materials. This would include investigating defects in the materials through calculations of the magnetic susceptibility, which quantity is directly correlated to defects in the materials. In addition, nuclear quadrupole moments and hexadecapole moments, which can be useful in determining the nuclear structure of the material, can be determined with NAR. Thus, NAR cannot only be used as a detection tool, but it also has the potential to be used in the laboratory to characterize intact materials imbedded in an apparatus. [4].

The unique advantage of this concept is that this technique does not rely on nuclear radiation detection. The device's design principle and main objective will be to detect and identify fissile material even if steps are taken by the would-be smugglers to completely shield the material as a radiation source. Therefore, a successful development of this device could have enormous potential for the detection and identification of concealed fissile materials.

In addition, NAR could be used to characterize unconventional superconductivity in $\mathrm{PuCOGa}_{5}$ [1]. Plutonium and other actinides behavior has not yet been fully revealed, including the discovery of the unconventional superconductivity in $\mathrm{PuCOGa}_{5}$ and the phonon dispersion present in plutonium. NMR has been used to study secondary elements such as Gallium to gain insight in the spin dynamics of this important material. This data showed that Gallium in this material was directly coupled to the Fermi surface due to local lattice distortions [5]. Previously, there was no evidence of any magnetic transitions or local moments. By using NAR techniques to directly measure electric field gradients, one can measure the local distortions in $\mathrm{PuCOGa}_{5}$ and provide further insight into plutonium systems.

NAR utilizes acoustic waves to penetrate into a material at the material's intrinsic nuclear and electronic frequencies and couple to the material's lattice. This creates new phonons with the same frequencies or energy as the acoustic waves. These phonons in turn perturb both the inter-nuclear distances and the overall charge distribution around nuclear sites. Charge perturbation creates electric field gradients, and the nuclear spins interact with the nuclear magnetic moments and nuclear electric quadrupoles, leading to splitting of spin energy levels in the presence of a static magnetic field, similarly to NMR. NAR and NMR are similar because they both induce shifts in the materials nuclear spin energy levels in the presence of an externally applied constant magnetic field, and the energy levels that are perturbed by an acoustic wave (NAR) or a radiofrequency (NMR) are affected in a similar fashion by both stimuli. NAR and NMR lead to energy shifts of different magnitudes. NAR makes it possible to perform noninvasive investigation of metals, where NMR is less effective.

The mechanism that allows us to evaluate metals with NAR is different from NMR. NAR coupling mechanisms occur because acoustic energy can couple to the material's nuclear spin system via various electric and magnetic interactions. [4] This includes the dynamic electric quadrupole interaction, in which the acoustic wave dramatically couples to the electric quadrupole moments of the nuclear spins; the magnetic dipole (or Alpher-Rubin) interaction [6], in which the acoustic waves couple to the magnetic moments of the nuclear spins; the hexadecapole interaction, in which the acoustic wave couples to the electric hexadecapole (16-pole) moments of the nuclear spins; and magnetic dipole-dipole interaction, in which acoustic wave couple to the materials via magnetic interactions among nuclear spins themselves. These various 
mechanisms couple to different multipole moments of the nuclear spins, each one leading to a different set of values for the observing interaction intensities, angular dependence, and line widths and line shapes.

In terms of signal intensities, empirically the most important nuclear spin-phonon interactions in NAR are the electric quadrupole and the magnetic dipole interaction (or Alpher-Rubin coupling). The electric quadrupole interaction is the most effective mechanism for coupling acoustic waves in nonconductors and in some conductors to nuclear spins with spin angular momentum I $>1 / 2$. On the other hand, the magnetic dipole (or Alpher-Rubin coupling) interaction is effective only in metals. This interaction depends on the electromagnetic interaction of conduction electrons with the acoustic waves in the presence of an externally applied static magnetic field. Since the magnetic dipole moment is coupled to the nuclear spins, this coupling occurs for nuclear spins with spin angular moments $I>1 / 2$ or $I=1 / 2$. These nuclear spin-phonon coupling mechanisms via NAR not only provide a mechanism to detect and distinguish different metals, which have different intrinsic electronic and nuclear frequencies, but can also provide valuable information about the structure, including impurities, of intact materials. In order to fully understand the advantages and limitations of this unique technology, we first need to demonstrate that NAR can be used in the laboratory under ideal conditions. While we are examining this technique, we will obtain useful information on an important question in actinide research: using NAR techniques to directly measure electric field gradients in $\mathrm{PuCOGa}_{5}$.

\section{Reference:}

[1] Curro NJ, Caldwell T, Bauer ED, Morales LA, Graf MJ, Bang Y, Balatsky AV, Thompson JD, Sarrao JL, NATURE 434 (7033): 622-625 , (2005)

[2] BBC newspaper, "German trio face arms charges", August 16, 2003, http://news.bbc.co.uk/2/hi/europe/3157283.stm

[3] website: http://www.dhushara.com/book/explod/nuclears/nuc.htm

[4] D. L. Bolef and R.K. Sundfors, Nuclear Acoustic Resonance, Academic Press,

Harcourt Brace \& Company, NY, 1993.

[5] Curro NJ, Morales L. Mater. Res. Soc. 2004, pp.53-8. Warrendale, PA, USA.

[6] P.A. Fedders, Physical Review B, 1974, 1740-1743, (1973)

\section{Acknowledgments:}

This work was performed under the auspices of the U.S. Department of Energy by the University of California, Lawrence Livermore National Laboratory, under contract \# W7405-ENG-48. 